\title{
Incertidumbre en docentes de los colegios de la ciudad de Pilar en contexto pandemia
}

\author{
Romina Diana Ramírez Torres
}

romidianatorres@gmail.com

\author{
Jeny Patricia Bobadilla Torres \\ bobadillajeny@gmail.com
}

Facultad de Humanidades y Ciencias de la Educación

Universidad Nacional de Pilar

Ciudad de Pilar - Paraguay.

\section{RESUMEN}

Se considera intolerancia hacia la incertidumbre, a la percepción y modo de respuesta de una persona ante situaciones inciertas o ambiguas. Por ello, este estudio se realiza con el objetivo de analizar el nivel de intolerancia a la incertidumbre de los docentes de los colegios de la ciudad de Pilar, año 2021. Adoptando como estrategia metodológica un enfoque cuantitativo y cualitativo, con diseño observacional, transversal y prospectivo para la descripción del fenómeno, los datos son analizados mediante procedimientos de estadística descriptiva, a partir de la administración y posterior aplicación del test, de Escala de Intolerancia hacia la Incertidumbre (IUS), de 27 ítems sobre incertidumbre, como factor de vulnerabilidad cognitiva que subyace al trastorno de ansiedad; y a su vez, el Inventario de Preocupación de Pensilvania, de 16 ítems, que evalúa la tendencia general o rasgo de preocuparse, variable que parece cumplir un importante rol en todos los procesos emocionales y en los trastornos de ansiedad y del estado de ánimo, a una muestra compuesta por 60 docentes de tres colegios de la ciudad de Pilar, concluyendo, que las situaciones inciertas y las ambigüedades de la vida, le causan estrés, además que hay muchas circunstancias que hacen que se preocupe.

Palabras clave: docencia; incertidumbre; pandemia; preocupación. 


\title{
Uncertainty in teachers of the schools of the city of Pilar in a pandemic context
}

\begin{abstract}
It is considered intolerance towards uncertainty, the perception and mode of response of a person to uncertain or ambiguous situations. For this reason, this study is carried out with the objective of analyzing the level of intolerance to uncertainty of the teachers of the schools of the city of Pilar, year 2021. Adopting as a methodological strategy a quantitative and qualitative approach, with an observational, cross-sectional and prospective for the description of the phenomenon, the data are analyzed using descriptive statistics procedures, from the administration and subsequent application of the test, the Intolerance towards Uncertainty Scale (IUS), of 27 items on uncertainty, as a factor of cognitive vulnerability underlying anxiety disorder; and in turn, the Pennsylvania Worry Inventory, with 16 items, which assesses the general tendency or trait to worry, a variable that seems to play an important role in all emotional processes and in anxiety and mood disorders, to a sample made up of 60 teachers from three schools in the city of Pilar, concluding that uncertain situations and ambiguities in life cause stress, and that there are many circumstances that make you worry.
\end{abstract}

Keywords: teaching; uncertainty; pandemic; worry.

Artículo recibido: 05 octubre. 2021 Aceptado para publicación: 02 noviembre 2021 Correspondencia: romidianatorres@gmail.com Conflictos de Interés: Ninguna que declarar 


\section{INTRODUCCIÓN}

La nueva enfermedad pandémica por coronavirus, llegó al Paraguay con el primer caso, el 7 de marzo de 2020. En respuesta, el Ejecutivo, por Decreto $\mathrm{N}^{\circ} 3442$ del 9 de marzo de 2020, dispuso "la implementación de acciones preventivas ante el riesgo de expansión del Coronavirus (COVID-19) al territorio Nacional". De acuerdo con este Decreto, prácticamente, la Cuarentena total comenzó el 11 de marzo (Ministerio de Salud Pública y Bienestar Social, 2020).

Cuando el 11 de marzo del 2020, la Organización Mundial de la Salud (OMS) declara al coronavirus COVID-19 como una Pandemia, inició un rotundo cambio del estilo de vida de las personas, de todas las edades, creencias, raza y estrato socioeconómico. Cada gobierno ha tomado decisiones y medidas para enfrentar a este virus, reestructurando los hábitos en los diferentes estratos sociales.

Las instituciones educativas se han cerrado en la mayoría de los países, obligando a los docentes a enseñar desde casa con la ayuda de los medios tecnológicos.

El propósito de este estudio es analizar el nivel de intolerancia hacia la incertidumbre de los docentes de tres colegios de la ciudad de Pilar, considerando a la preocupación como una variable preponderante para el curso y avance de trastornos de ansiedad, por ello se plantea: Identificar las condiciones sociodemográficas de los docentes de los colegios de la ciudad de Pilar, a partir de cuestionario básico que contenga los datos de la muestra estudiada. Así también se pretende; reconocer el nivel de intolerancia hacia la incertidumbre de los docentes de los colegios, haciendo uso de instrumentos psicológicos estandarizados, cuya finalidad es medir la tendencia o rasgo de incertidumbre de los docentes de tres colegios de la ciudad de Pilar, por consiguiente, se orienta a determinar el grado de preocupación de la misma muestra, respetando los criterios establecidos en el Inventario de Preocupación de Pensilvania, implementado como herramienta de análisis y evaluación en este estudio.

Se ha consultado bibliografías, opiniones y posturas de varios expertos, y resultados de algunas investigaciones sobre la ansiedad, los factores de vulnerabilidad durante la pandemia, los protocolos establecidos, junto con las normas de bioseguridad como parte preventiva de propagación de la enfermedad, han aumentado el nivel de incertidumbre de los docentes. 
Por tanto, se evidencia, que, por decisión de los gobiernos, se implementa la educación virtual, con algunas incertidumbres, situación que resulta complicada para algunos, a otros les agrada y existen los que tienen la esperanza de que esta oportunidad sea aprovechada por las autoridades para iniciar la reforma educativa tan ansiada por la comunidad educativa (Medina, 2020).

Entre algunos de los acuerdos, está El Plan de educación en tiempos de pandemia "Tu Escuela en Casa", que prevé un alcance del servicio educativo para 1.463 .620 estudiantes matriculados en la educación básica en instituciones de gestión oficial, privada y privada subvencionada, en la que se busca que al menos el $90 \%$ de los docentes en servicio del sector oficial desarrollen competencias en el uso de las TIC durante el proceso educativo y la interacción con los estudiantes (Wehrle Martínez, 2020). En cuanto a la relación con las familias, se espera que los padres/tutores de hogares compuesto por al menos una persona en edad escolar, de 5 a 17 años de edad, acompañen el proceso de aprendizaje de sus hijos y menores a su cargo, en vista del aumento de las responsabilidades y exigencias educativas (Sanabria Benítez., 2020).

Es relevante, la atención del impacto de la pandemia en la salud mental, no solo para mejorar la salud sino también para evitar otros problemas sociales, tales como la estigmatización de personas, la falta de adherencia a medidas de prevención, y el duelo frente a la pérdida de seres queridos. En definitiva, estos factores tienen un rol fundamental para afrontar la pandemia de manera integral. Atendiendo el impacto social y consecuencias que el COVID-19 produce a nivel global, este trabajo realiza un análisis de los signos y síntomas de los trastornos de ansiedad; la incertidumbre y preocupación percibida por docentes de colegios del área urbana y periurbana específicamente.

\section{Antecedentes.}

Taylor refiere que en situaciones de pandemia "es esperable que las personas sientan miedo, ansiedad, angustia, irritabilidad, estrés y enojo, recuerdo de traumas, dificultades para la concentración y/o problemas en el sueño" (Johnson, Saletti Cuesta, \& Tumas, 2020).

Trabajos recientes advierten sobre las consecuencias en la salud mental que el COVID19 y el aislamiento social provocan en la población" porque "aún no existen vacunas ni tratamientos efectivos, se desconoce la duración de las medidas de aislamiento, y se estima un alto impacto económico, social y sanitario, lo que aumenta aún más la 
incertidumbre" Es más, "se advierte un impacto en la salud mental, expresado en sentimientos de miedo, incertidumbre y angustia, propios de un sentido de ruptura en la cotidianidad y pérdida de previsibilidad que supone esta pandemia, especialmente el aislamiento que conlleva"

Cabe destacar que los sentimientos de Incertidumbre, Miedo, Responsabilidad y Angustia, que constituyen las expresiones más relevantes entre las personas participantes, se manifestaron con mayor preeminencia entre las mujeres.

El sentimiento de Responsabilidad incluye la importancia de los cuidados y la responsabilidad individual y colectiva para prevenir el COVID-19. La creciente precaución frente a la enfermedad y la prevención, son aspectos claves que nombran algunas personas, independientemente de su nivel educativo en ambas tomas de datos: "Respeto. Conocimiento. Cuidado" (Ola 1, mujer, 54 años). La forma en que el virus se propaga, exige un mayor registro del propio cuerpo, por ejemplo, con en el lavado de manos y también respecto al cuerpo de las otras personas, especialmente en lo relacionado con evitar el contacto y mantener la distancia. Ello se traduce, por un lado, en respeto y cuidado hacia los demás, especialmente de quienes pueden estar en una posición más vulnerable frente al virus, pero también en el cuestionamiento de las conductas de algunas personas frente a las medidas de prevención, que son calificadas como irresponsabilidad, tal como da cuenta esta expresión: La incapacidad de mucha gente para respetar normas por un bien común superior. Egoísmo (Ola 1, mujer, 36 años). Este sentimiento de responsabilidad pone en manifiesto la incorporación de los discursos preventivos: Ser responsable, tomar medidas de prevención, aislamiento social (Ola 1, mujer, 37 años); Prevención, prudencia, responsabilidad (Ola 1, mujer, 55 años). Cabe señalar también que la brecha advertida en la primera ola de la encuesta entre mujeres y varones respecto al sentimiento de Responsabilidad, se reduce en la segunda ola. Respecto a la percepción de confort en su hogar ante la situación de aislamiento social, observamos que quienes perciben mayor confort mencionan más la importancia de la responsabilidad y los cuidados frente a la pandemia. (Johnson, Saletti Cuesta, \& Tumas, 2020).

La Angustia aparece e incluye sentimientos que van desde la tristeza, el aburrimiento, hasta la depresión. En ocasiones este sentimiento se vincula a otras personas, como por ejemplo los seres queridos, o hacia personas en situación de exclusión social: Angustia por familiares que deben ir a trabajar y por mi nieta que está en India y no puede 
volver (Ola 1, mujer, 71 años); Tristeza por los más desfavorecidos (Ola 1, hombre, 51 años) (Johnson, Saletti Cuesta, \& Tumas, 2020).

La Soledad aparece vinculada al encierro y al aislamiento. En palabras de una participante: Aislamiento indefinido. Extrañar la rutina y a la familia (Ola 2, mujer, 19 años). Por otro lado, esta pandemia genera un sentimiento de Vulnerabilidad que se relaciona con la indefensión, la fragilidad, la inseguridad, la posibilidad de morir debido a una probable infección y también con la impotencia que ello genera. Ambos sentimientos estuvieron más presentes entre mujeres en ambos momentos, aunque la brecha es menor en la segunda ola. También se reducen en la segunda ola las brechas de género en las categorías de Angustia y Vulnerabilidad. Con menor frecuencia, aparecen los sentimientos de Descontrol expresado como locura, crisis, caos y paranoia generados por la pandemia y de Enojo, en este caso vinculado a expresiones de bronca y hartazgo. (Johnson, Saletti Cuesta, \& Tumas, 2020).

La característica principal del Trastorno de Ansiedad Generalizada (TAG) es tener una preocupación y ansiedad excesivas (expectativa aprensiva o aprensión ansiosa), persistentes (más de la mitad de los días durante al menos 6 meses) y difíciles de controlar sobre un número de acontecimientos o actividades tales como el rendimiento laboral o escolar. Que la preocupación y ansiedad son excesivas significa que su intensidad, duración o frecuencia son desproporcionadas con relación a la probabilidad o impacto real del evento temido (Bados, 2009).

Como parte de esa característica, se encuentra la "intolerancia a la incertidumbre", el cual, es la tendencia excesiva del individuo a considerar como inaceptable que pueda suceder un evento negativo, por más pequeña que sea la probabilidad de que ocurra (Dugas, Gosselin, \& Ladouceur, 2001). Resultado de un conjunto de creencias negativas sobre la incertidumbre, disposiciones y sus implicancias, característica del sujeto que la padece, que (Koerner \& Dugas, 2008) citados en (Rodríguez de Behrends \& Brenilla, 2015).

A su vez, "la preocupación", descrita como un tipo de pensamiento repetitivo negativo, una experiencia común a todos los individuos, constructiva y en ocasiones necesaria para tratar de resolver problemas. La preocupación cumple con una función de alerta y de adaptación, pero cuando no cumple con dicha función genera elevados niveles de malestar y se vuelve patológica (Ferrer, 2002). En cuanto a la preocupación patológica, el componente principal del Trastorno de Ansiedad Generalizada (TAG), se caracteriza por 
la presencia de preocupación "ansiosa" que el paciente refiere como incontrolable y muy frecuente (Padros Blazquez, Gonzalez Betanzos, Martinez Medina, \& Wagner, 2018). La preocupación le resulta difícil de controlar a la persona, así como al menos tres síntomas físicos de una lista de seis (inquietud o impaciencia, fatigabilidad fácil, dificultad para concentrarse o tener la mente en blanco, irritabilidad, tensión muscular y alteraciones del sueño)". La preocupación es definida por el equipo de investigación del Estado de Pensilvania (Estados Unidos) como una actividad lingüística verbal, relativamente incontrolable, acompañada de un estado afectivo negativo y que puede emplearse como una conducta cognitiva evitativa, que se caracteriza por reducir o inhibir las actividades de tipo imaginario y fisiológico de las personas con ansiedad. El proceso de preocupación representa un intento de solución mental de problemas sobre un tema cuyo resultado es impredecible (Borkovec \& Inz, 1990) citados en (Alemán Herrera, Herrera Padilla, \& Martínez Torres, 2019).

La intolerancia hacia la incertidumbre forma parte de un constructo más amplio denominado intolerancia hacia la ambigüedad (Frenkel Brunswik, 1948), que ha recibido múltiples interpretaciones dependiendo del matiz y sentido con que se utilice el término. En general, puede decirse que la intolerancia hacia la ambigüedad hace referencia "al modo en que una persona (o grupo) percibe y procesa la información sobre situaciones ambiguas cuando se enfrenta con una variedad de señales no familiares, complejas o incongruentes" Furnham, 1994, p. 403 citados en (González Rodríguez, Cubas León, Rovella, \& Darias Herrera, 2006). La persona con alta intolerancia hacia la ambigüedad experimenta estrés, reacciona prematuramente y evita estímulos ambiguos. En el otro extremo del continuo, sin embargo, una persona con baja intolerancia hacia la ambigüedad percibe las situaciones ambiguas como deseables, desafiantes e interesantes y no niega ni distorsiona su complejidad o incongruencia.

\section{ESTRATEGIAS METODOLÓGICAS O MATERIALES Y MÉTODOS}

El trabajo de investigación corresponde a la metodología de tipo descriptivo, comprende la descripción, registro, análisis e interpretación de la naturaleza actual, y la composición o proceso de los fenómenos. El enfoque se hace sobre conclusiones dominantes o sobre grupo de personas, grupo o cosas, se conduce o funciona en presente (Tamayo y Tamayo, 2003). De enfoque cuantitativo, el cual parte de una idea que va acotándose y, una vez delimitada, se derivan objetivos y preguntas de investigación, se revisa la literatura y se 
construye un marco o una perspectiva teórica. De corte transversal que analiza datos de variables recopiladas en un periodo de tiempo sobre una población muestra o subconjunto predefinido (Hernández Sampieri, Fernández Collado, \& Baptista Lucio, 2014).

Como estrategia de acceso a la información, se realiza charlas sobre la ansiedad, la preocupación, el estrés, depresión y el manejo de las emociones, de aproximadamente una hora de duración, en la cual se informa acerca de los temas ya descritos en los objetivos, y posterior al mismo se administra y aplica el test sobre, Escala de Intolerancia hacia la Incertidumbre (IUS), dicha escala es de gran utilidad en la detección de trastorno de ansiedad generalizada, al considerar la intolerancia hacia la incertidumbre como un factor de vulnerabilidad cognitiva que subyace a dicho trastorno (Dugas y Ladouceur, 1998; Dugas y cols., 1998; Dugas y cols., 2005). La escala consta de 27 ítems sobre incertidumbre, reacciones emocionales y conductuales ante situaciones ambiguas, implicaciones de inseguridad e intentos de controlar el futuro (González Rodríguez, Cubas León, Rovella, \& Darias Herrera, 2006).

Además, siguiendo con los propósitos planteados en este estudio se aplica, el Inventario de Preocupación de Pensilvania (Penn State Worry Questionnaire) (PSWQ; Meyer, Miller, Metzger y Borkovec, 1990) el cual, tiene por objetivo evaluar la tendencia general a preocuparse o rasgo de preocupación, variable que parece cumplir un importante rol en todos los procesos emocionales y en los trastornos de ansiedad y del estado de ánimo, pero que es especialmente relevante en el Trastorno de Ansiedad Generalizada, cuya principal característica diagnóstica es precisamente una preocupación excesiva e incontrolable (Alemán Herrera, Herrera Padilla, \& Martínez Torres, 2019).

Está compuesto por 16 ítems, que evalúan mediante escalas de intervalo (desde 1, "nada", a 5, "mucho"; rango de puntuación = 16-80). La versión original inglesa presentaba cinco ítems en sentido inverso que fueron invertidos en la versión española para personas mayores (Nuevo, Montorio y Ruiz, 2002), de acuerdo con los resultados de un estudio piloto en el que se presentaron problemas de comprensión con esos ítems y con diversos trabajos con la versión inglesa en los que se había encontrado con estos cinco ítems se agrupaban en un factor independiente y se recomendaba redactarlos en positivo o eliminarlos. Además, la redacción de los ítems fue evaluada en el estudio piloto para maximizar su aplicabilidad en personas de edad avanzada (Alemán Herrera, Herrera Padilla, \& Martínez Torres, 2019). 
Tabla 1. Operacionalización de Variables

\begin{tabular}{lll}
\hline \multicolumn{1}{c}{ Variables } & \multicolumn{1}{c}{ Definición conceptual } & Indicadores \\
\hline $\begin{array}{l}\text { Intolerancia a la } \\
\text { incertidumbre }\end{array}$ & $\begin{array}{l}\text { La intolerancia a la incertidumbre, es la } \\
\text { tendencia excesiva del individuo a } \\
\text { considerar como inaceptable que pueda } \\
\text { suceder un evento negativo, por más } \\
\text { pequeña que sea la probabilidad de que } \\
\text { ocurra }\end{array}$ & $\begin{array}{l}\text { Poco, nada. } \\
\text { Moderadamente } \\
\text { Extremadamente }\end{array}$ \\
\hline $\begin{array}{l}\text { Nivel de } \\
\text { preocupación }\end{array}$ & $\begin{array}{l}\text { Estado de ocupación del pensamiento } \\
\text { determinadas formas de preocupación } \\
\text { como próximas a la angustia. }\end{array}$ & Nada, algo. \\
\hline Condiciones & $\begin{array}{l}\text { Incluye las características de las } \\
\text { situaciones manifiestas en los docentes } \\
\text { de la muestra de estudio. }\end{array}$ & $\begin{array}{l}\text { Edad, } \\
\text { Sexo } \\
\text { sociodemográfica civil }\end{array}$ \\
\hline
\end{tabular}

Fuente: Elaboración propia.

\section{RESULTADOS Y DISCUSIÓN}

La pandemia del COVID - 19 ha creado una dinámica diferente en la relación de docentes - escolares y sus familias, sin embargo, esto es asimilado de formas diferentes dependiendo del nivel socio económico de cada persona, principalmente debido al acceso tecnológico, es así que, en respuesta al objetivo de Identificar las condiciones sociodemográficas de los docentes de los colegios de la ciudad de Pilar, año 2021. Se observa que la población y muestra se describe en cuanto sigue:

\section{Tabla 2. Unidad de Estudio}

\begin{tabular}{|c|c|c|}
\hline Fuentes & $\begin{array}{c}\text { Muestra } \\
\text { Sexo y Edad }\end{array}$ & Instrumento \\
\hline $\begin{array}{l}\text { Docentes del } \\
\text { Colegio Nacional } \\
\text { San Lorenzo }\end{array}$ & $\begin{array}{c}30(25-\mathrm{F})(5-\mathrm{M}) 30-58 \\
\text { años }\end{array}$ & $\begin{array}{llll}\text { Escala de } & \text { Intolerancia } & \text { hacia } & \text { la } \\
\text { Incertidumbre (IUS) } & & \\
\text { Inventario de } & \text { Preocupación } & \text { de } \\
\text { Pensilvania. } & & & \\
\end{array}$ \\
\hline $\begin{array}{l}\text { Docentes del } \\
\text { Colegio Nacional } \\
\text { Pilar. }\end{array}$ & $\begin{array}{c}19(15-\mathrm{F})(4-\mathrm{M}) \quad 30-58 \\
\text { años }\end{array}$ & $\begin{array}{l}\text { Escala de Intolerancia hacia la } \\
\text { Incertidumbre (IUS) } \\
\text { Inventario de Preocupación de } \\
\text { Pensilvania. }\end{array}$ \\
\hline $\begin{array}{l}\text { Docentes } \text { del } \\
\text { Colegio Privado } \\
\text { Subvencionado } \\
\text { "Virgen } \\
\text { Fátima". }\end{array}$ & $\begin{array}{c}11(9-\mathrm{F})(2-\mathrm{M}) \quad 30-58 \\
\text { años }\end{array}$ & $\begin{array}{l}\text { Escala de Intolerancia hacia la } \\
\text { Incertidumbre (IUS) } \\
\text { Inventario de Preocupación de } \\
\text { Pensilvania. }\end{array}$ \\
\hline TOTAL & $\begin{array}{c}60 \text { (49-F) (11-M) } 30 \text { - } \\
58 \text { años }\end{array}$ & Personas \\
\hline
\end{tabular}

Fuente: Elaboración propia. 
Con respecto al segundo objetivo, de: Reconocer el nivel de intolerancia hacia la incertidumbre de los docentes de los colegios de la ciudad de Pilar, año 2021; a partir de la aplicación de la Escala de Intolerancia hacia la Incertidumbre (IUS), a una muestra compuesta por 60 docentes de los tres colegios mencionados, en los turnos mañana y tarde. Se obtuvieron los siguientes resultados, de acuerdo a los indicadores propios de la escala.

En el ítem, La incertidumbre me impide tener una opinión firme de la escala de tolerancia hacia la incertidumbre, de los 60 docentes de colegios de la muestra, 31 (52\%) respondió extremadamente; 20 (33\%) docentes moderadamente; en tanto que, $5(8 \%)$ de los mismos indicó que nada y 4 (7\%) docentes como nada característico.

Ante el indicador de inseguridad y sensación de desorganización, 30 (50\%) docentes de la muestra perciben como extremadamente característico; 20 (33\%) docentes manifiestan moderadamente característico; mientras que el 12\% del total de la muestra representado por 7 docentes, como poco, y 5\% nada característico sobre sí mismo.

De los 60 docentes de la muestra, 35 (59\%) refiere que extremadamente la incertidumbre hace intolerable la vida; 15 (25\%) docentes moderadamente; así también 8 docentes representado por el $13 \%$ manifiesta que poco y 2 (3\%) como nada característico.

En relación, a que si es injusto no tener garantías de que las cosas vayan a salir bien en la vida; 25 docentes representado por el $42 \%$ de la muestra manifestó como moderadamente característico, y 15 (25\%) docentes distribuidos cada uno entre poco y nada característico. Analizando la variable de, no puede estar tranquilo/a mientras no sepa lo que va a suceder al día siguiente, 35 docentes representado por el $64 \%$ de la muestra refirió como nada característico, $10(18 \%)$ docentes como poco; en tanto que, moderadamente y extremadamente característicos representado por el 9\% del total de la muestra cada uno. Con la percepción de que la incertidumbre produce inquietud, ansiedad o estrés, 35 de los 60 docentes de la muestra, indicó como extremadamente característico, 15 (25\%) moderadamente y $5(8 \%)$ docentes como poco y nada característico.

De acuerdo al ítem, los imprevistos me molestan mucho, de los 60 (100\%) docentes, 35 (59\%) manifiestan como nada característico dentro de la escala de intolerancia hacia la incertidumbre; 15 (25\%) docentes como poco característico y en moderada $\mathrm{y}$ extremadamente $5(15 \%)$ docentes en cada escala. 
Dentro de la escala moderadamente característico 35 docentes representado por el $59 \%$ de la muestra, percibe que es frustrante no tener toda la información que necesita, no obstante, 15 docentes representado por el 25\%, lo indica como nada característico.

Se observa que, dentro de la escala del instrumento psicológico aplicado, 20 (33\%) docentes siente que moderadamente la incertidumbre le impide disfrutar plenamente de la vida; 19 (32\%) docentes como poco y 11 (18\%) docentes como nada característico.

Mediante la Escala de Intolerancia hacia la Incertidumbre, se reconoce que 42 (70\%) docentes cree que se debería prever todo para evitar las sorpresas, mientras que, 8 (13\%) docentes representado por $13 \%$ de la muestra indicó moderadamente; así también se evidencia que $6(10 \%)$ docentes refieren como poco y $4(7 \%)$ docentes como nada característico. Por consiguiente, 35 (59\%) docentes, indicó dentro de la escala de nada característico que un pequeño imprevisto puede arruinarlo todo, incluso con la mejor de las planificaciones, 15 (25\%) docentes como moderadamente y en las escalas moderada y extremadamente la distribución de $5(8 \%)$ docentes.

En relación, al ítem de que, cuando llega el momento de actuar, la incertidumbre me paraliza, de los 60 docentes de la muestra, 25 indicó como nada característico, 18 (30\%) moderada, $12(20 \%)$ docentes extremadamente y $5(8 \%)$ docentes dentro de la escala de poco característico.

A través de la escala, se reconoce a 35 (59\%) docentes dentro de nada característico, 15 (25\%) docentes en poco característico; 5 (8\%) docentes en moderada y extremadamente, en el criterio, de que estar inseguro, implica no poder figurar entre los mejores. Del mismo instrumento, se deduce que, 35 (59\%) docentes menciona que dentro de la escala moderadamente característico cuando está indeciso/a no puede seguir adelante; 15 (25\%) docentes dentro de la escala de nada y seguidos de $5(8 \%)$ docentes en poco y extremadamente característico oportunamente.

En función al criterio de que, cuando estoy indeciso/a no puedo funcionar bien, 35 (59\%) docentes indica como nada característico; 15 (25\%) docentes en el rango de poco y 5 $(8 \%)$ docentes dentro de moderada y extremadamente característico. En respuesta al indicador de que, "a diferencia de mí, los demás siempre parecen saber hacia dónde dirigen sus vidas", $35(59 \%)$ se identificó como poco característico, 15 (25\%) moderadamente, $5(8 \%)$ docentes en nada, y así también en extremadamente característico respectivamente. 
Además, se observa que, 38 docentes representado por el $63 \%$ de la muestra analizada, refiere dentro de la escala de extremadamente que la incertidumbre lo hace vulnerable, infeliz o triste; 12 (20\%) docentes moderada, 6 (10\%) docentes nada y 4 (7\%) docentes como poco característico.

De la misma forma, respondiendo al criterio de que, "quiero saber siempre qué me depara el futuro", 25 (41\%) docentes se encuentra dentro de la escala moderadamente característico, 15 (25\%) docentes en extremadamente; 13 (22\%) docentes en poco y 7 (12\%) en nada característico. De los 60 (100\%) docentes de los colegios de la ciudad de Pilar, tomadas como muestra, 35 (59\%) menciona dentro de la escala de extremadamente característico, no soportar que lo cojan por sorpresa; 15 (25\%) docentes como moderadamente característico, y en poco y nada característico 5 (8\%) docentes respectivamente.

En el aspecto actitudinal, 37 (62\%) docentes indicaron en la escala de nada característico, al indicador de que la más mínima duda lo puede impedir actuar; a su vez, se observa a $13(22 \%)$ docentes en poco característico, 8 (13\%) docentes en moderada y $2(3 \%)$ docentes en extremadamente característico. Pensando en que, si tendría que ser capaz de organizar todo de antemano, 35 docentes de la muestra representado por el 59\% del total de la muestra se encuentra dentro de la escala moderadamente característico, 15 docentes indicaron que nada característico, y 5 docentes en las escalas poco y en extremadamente característico respectivamente.

En cuanto al criterio de sensación de que, la incertidumbre produce falta de confianza en sí mismo, 35 docentes mencionaron que extremadamente, 15 moderadamente, como poco característico 8 docentes y, 2 docentes en la escala de nada característico.

Respondiendo, a la expresión "No entiendo cómo otras personas parecen tan seguras y decididas acerca de su futuro", 35 docentes se encuentran dentro de la escala de nada característico, 15 en poco, 8 en moderada y 2 en extremadamente.

Se identifica que, en el indicador, "la incertidumbre me impide dormir bien", 25 docentes se encuentran, dentro de la escala de nada y moderadamente característico de mí, 8 docentes en la escala de poco y 2 docentes en extremadamente característico respectivamente. Como variable preponderante de la escala de intolerancia hacia la incertidumbre, se menciona que, debo alejarme de toda situación incierta, hallándose en 
la escala de moderadamente característico a 35 docentes, 15 en el de poco característico y, 5 docentes en las escalas de nada y extremadamente característicos respectivamente. A partir del indicador, las ambigüedades de la vida me causan estrés; se identifica a 25 (42\%) docentes dentro de la escala de extremadamente característico, 15 (25\%) docentes en las escalas nada y moderada respectivamente, a su vez $5(8 \%)$ docentes como poco característico. Por último, en el criterio que refiere, no soporto estar indeciso/a acerca de mi futuro, $25(42 \%)$ docentes se encuentran dentro de la escala de moderadamente característico, 20 (33\%) docentes en poco, 10 (17\%) en nada característico y 5 (8\%) docentes en extremadamente respectivamente.

Respondiendo al último objetivo de, Determinar el grado de preocupación presente en los docentes de los colegios, se exponen los resultados del instrumento utilizado:

\section{Tabla 3. Inventario de Preocupación de Pensilvania.}

\begin{tabular}{|c|c|}
\hline $\begin{array}{l}\text { El reactivo más } \\
\text { recurrente en el } \\
\text { Colegio Nacional } \\
\text { San Lorenzo. }\end{array}$ & $\begin{array}{l}\text { De acuerdo a los resultados que se obtuvieron con este grupo de } \\
\text { docentes, en el primer punto el } 65 \% \text { destaca un elevado grado de } \\
\text { preocupación por no contar con tiempo suficiente para realizar sus } \\
\text { cosas. } \\
\text { Así también el } 60 \% \text { mencionaron que existen muchas circunstancias } \\
\text { que hacen que se preocupen, igualmente el } 55 \% \text { le resulta difícil dejar } \\
\text { de lado las preocupaciones y el mismo porcentaje, un } 55 \% \text { señalan que, } \\
\text { aunque no haya más que puedan hacer sigue preocupándose por ello. }\end{array}$ \\
\hline $\begin{array}{l}\text { El reactivo más } \\
\text { recurrente en el } \\
\text { Colegio Nacional } \\
\text { Pilar }\end{array}$ & $\begin{array}{l}\text { Según las respuestas que puntuaron los docentes de esta institución, } \\
\text { entre las más relevantes evidencian que el } 50 \% \text { siente preocupación si } \\
\text { no tiene tiempo suficiente para hacerlo todo, así como al } 50 \% \text { le resulta } \\
\text { difícil dejar sus preocupaciones de lado. } \\
\text { En otras de las afirmaciones el } 55 \% \text { insinúa que cuando comienza a } \\
\text { preocuparse por algo ya no puede parar y de modo similar el } 60 \% \text { se } \\
\text { preocupa por un proyecto hasta que esté acabado. } \\
\text { Cabe destacar que } 80 \% \text {, casi el total de la población indico que cuando } \\
\text { está bajo tensión tiende a preocuparse demasiado. }\end{array}$ \\
\hline $\begin{array}{l}\text { El reactivo más } \\
\text { recurrente en el } \\
\text { Colegio Privado } \\
\text { Subvencionado } \\
\text { Virgen de Fátima }\end{array}$ & $\begin{array}{l}\text { En comparación con los resultados de las otras instituciones las } \\
\text { respuestas de la mayoría de los ítems del inventario demuestran que los } \\
\text { docentes de este Colegio tienen un elevado grado de preocupación. } \\
\text { Así lo refleja el } 80 \% \text {, que se preocupa si no tiene tiempo suficiente } \\
\text { llevar a cabo sus tareas. Un } 70 \% \text { suele preocuparse por las cosas del } \\
\text { mismo modo que el } 65 \% \text { afirma la existencia de muchas circunstancias } \\
\text { que le preocupan y un } 60 \% \text { expresa que tiende a preocuparse mucho } \\
\text { cuando está bajo tensión. } \\
\text { Entre las aseveraciones más valoradas destaca que el } 70 \% \text { les resulta } \\
\text { difícil dejar de lado las preocupaciones de lado y aunque no haya más } \\
\text { que pueda hacer sigue preocupándose por ello. De manera similar un } \\
80 \% \text { menciona que no puede parar una vez que comienza a preocuparse } \\
\text { por algo y finalmente el } 90 \% \text { se preocupa por un proyecto hasta que } \\
\text { esté acabado }\end{array}$ \\
\hline
\end{tabular}

Fuente: Elaboración propia. 
En el Colegio San Lorenzo, los ítems más recurrentes, se determina en cuanto sigue:

\begin{tabular}{lll}
\hline Se preocupa si no tiene tiempo suficiente para hacerlo todo & 13 docentes & $65 \%$ \\
\hline Suele preocuparse por las cosas & 10 docentes & $50 \%$ \\
\hline Hay muchas circunstancias que hacen que se preocupe & 12 docentes & $60 \%$ \\
\hline $\begin{array}{ll}\text { Cuando está bajo tensión tiende a preocuparse mucho } \\
\text { le resulta difícil dejar de lado las preocupaciones }\end{array}$ & 11 docentes & $45 \%$ \\
\hline $\begin{array}{l}\text { Aunque no haya más que pueda hacer sigue preocupándose } \\
\text { por ello }\end{array}$ & $55 \%$ \\
\hline $\begin{array}{l}\text { Una vez que comienza a preocuparse por algo, ya no puede } \\
\text { parar }\end{array}$ & 5 docentes & \\
\hline Se preocupa por un proyecto hasta que esté acabado & 8 docentes & $40 \%$ \\
\hline
\end{tabular}

Fuente: Elaboración propia.

En el Colegio Nacional Pilar, los ítems más recurrentes, se determina en cuanto sigue:

\begin{tabular}{|c|c|c|}
\hline Se preocupa si no tiene tiempo suficiente para hacerlo todo & 10 docentes & $50 \%$ \\
\hline Suele preocuparse por las cosas & 9 docentes & $45 \%$ \\
\hline Hay muchas circunstancias que hacen que se preocupe & 9 docentes & $45 \%$ \\
\hline Cuando está bajo tensión tiende a preocuparse mucho & 16 docentes & $80 \%$ \\
\hline le resulta difícil dejar de lado las preocupaciones & 10 docentes & $50 \%$ \\
\hline $\begin{array}{l}\text { Aunque no haya más que pueda hacer por algo sigue } \\
\text { preocupándose por ello }\end{array}$ & 8 docentes & $40 \%$ \\
\hline $\begin{array}{l}\text { Una vez que comienza a preocuparse por algo, ya no puede } \\
\text { parar }\end{array}$ & 11 docentes & $55 \%$ \\
\hline Se preocupa por un proyecto hasta que esté acabado & 12 docentes & $60 \%$ \\
\hline
\end{tabular}

Fuente: Elaboración propia.

En el Colegio Virgen De Fátima, los ítems más recurrentes, se determina en cuanto sigue:

\begin{tabular}{lll}
\hline Se preocupa si no tiene tiempo suficiente para hacerlo todo & 16 docentes & $80 \%$ \\
\hline Suele preocuparse por las cosas & 14 docentes & $70 \%$ \\
\hline Hay muchas circunstancias que hacen que se preocupe & 13 docentes & $65 \%$ \\
\hline Cuando está bajo tensión tiende a preocuparse mucho & 12 docentes & $60 \%$ \\
\hline Le resulta difícil dejar de lado las preocupaciones de lado & 14 docentes & $70 \%$ \\
\hline $\begin{array}{l}\text { Aunque no haya más que pueda hacer sigue preocupándose } \\
\text { por ello }\end{array}$ & 14 docentes & $70 \%$ \\
\hline $\begin{array}{l}\text { Una vez que comienza a preocuparse por algo ya no puede } \\
\text { parar }\end{array}$ & 16 docentes & $80 \%$ \\
\hline Se preocupa por un proyecto hasta que esté acabado & 18 docentes & $90 \%$ \\
\hline
\end{tabular}

Fuente: Elaboración propia. 


\begin{tabular}{lc}
\hline \multicolumn{1}{c}{ Ítems con mayor puntuación } & $\begin{array}{c}\text { Docentes que puntuaron } \\
\text { Bastante-Mucho }\end{array}$ \\
\hline $\begin{array}{l}\text { Se preocupa si no tiene tiempo suficiente para } \\
\text { hacerlo todo }\end{array}$ & $40-67 \%$ \\
\hline $\begin{array}{l}\text { Suele preocuparse por las cosas } \\
\text { Hay muchas circunstancias que hacen que se } \\
\text { preocupe }\end{array}$ & $30-50 \%$ \\
\hline $\begin{array}{l}\text { Cuando está bajo tensión tiende a preocuparse } \\
\text { mucho }\end{array}$ & $38-63 \%$ \\
\hline Le resulta difícil dejar de lado las preocupaciones & $34-57 \%$ \\
\hline $\begin{array}{l}\text { Aunque no haya más que pueda hacer sigue } \\
\text { preocupándose por ello }\end{array}$ & $32-54 \%$ \\
\hline $\begin{array}{l}\text { Una vez que comienza a preocuparse por algo, ya no } \\
\text { puede parar }\end{array}$ & $40-67 \%$ \\
\hline Se preocupa por un proyecto hasta que está acabado \\
\hline
\end{tabular}

\section{Fuente: Elaboración propia.}

Según los resultados del Inventario de Preocupación de Pennsylvania, aplicado a 60 docentes de tres instituciones educativas de la ciudad de Pilar, en el mes de octubre de 2021 (Colegio Nacional San Lorenzo, Colegio Nacional de Pilar y Colegio Privado Subvencionado Virgen de Fátima) arrojaron un elevado grado de preocupación que están reflejados en los siguientes ítems:

El $67 \%$ de los docentes afirman estar bastante preocupados al no contar con el tiempo suficiente para hacer sus cosas. El 54\% que corresponde a la mitad de la población respondieron que constantemente se preocupan por las cosas, en otra de las afirmaciones el 50\% indicaron que existen muchas circunstancias que hacen que se preocupe, como así también el 63\% señala que suelen preocuparse mucho cuando está bajo tensión.

Al 57\% le resulta difícil dejar de lado las preocupaciones, 57\% Aunque no haya más que pueda hacer sigue preocupándose por ello; $54 \%$ Una vez que comienza a preocuparse por algo, ya no puede parar; $67 \%$ Se preocupa por un proyecto hasta que está acabado.

\section{CONCLUSIÓN O CONSIDERACIONES FINALES}

Con base a los datos obtenidos en los instrumentos psicológicos administrados, se responde al objetivo principal de la investigación, quedando como resultante del análisis que el mayor número de docentes refiere que extremadamente la incertidumbre hace intolerable la vida; con la percepción de que la incertidumbre produce inquietud, ansiedad 
o estrés. Se observa que, de los 60 docentes de la muestra, 20, siente que moderadamente la incertidumbre le impide disfrutar plenamente de la vida.

Además, se observa que, 38 docentes representado por el $63 \%$ de la muestra analizada, refiere dentro de la escala de extremadamente que la incertidumbre lo hace vulnerable, infeliz o triste; evidenciando un alto porcentaje de docentes reflejados con el indicador, las ambigüedades de la vida me causan estrés; se reconoce que las áreas más comunes de preocupación suelen hacer referencia a circunstancias de la vida diaria; en las que son habituales temas como la familia, los amigos, las relaciones interpersonales en general, el dinero, el trabajo, los estudios, el manejo de la casa y la salud propia y de otros.

Conviene tener en cuenta todos los factores desencadenantes, los más vulnerables, de riesgo o precipitantes para indicar el mejor tratamiento que se ajuste a los requerimientos de la persona, atendiendo los elementos o mecanismos de confrontación que dispone, por ello se identifica las condiciones sociodemográficas, en lo que respecta a sexo, el mayor número corresponde al sexo femenino, de estado civil soltera de entre los 30 - 58 años de edad, más de la mitad enseña en ambos turnos (mañana y tarde).

Mediante la aplicación del Inventario de Preocupación de Pensilvania, se logra determinar que un mayor porcentaje de docentes, manifiesta una tendencia considerable de sensación de preocupación, al no contar con el tiempo suficiente para hacer sus cosas, además de la existencia de muchas circunstancias que hacen que se preocupe, entre los que mencionan, las capacitaciones, la adopción de las nuevas herramientas pedagógicas, participación de talleres de innovación de diseños metodológicos curricular, y el factor afectivo en la dimensión familiar, sumado a las exigencias y compromisos, que lo lleva a estar bajo tensión.

\section{LISTA DE REFERENCIAS}

Alemán Herrera, C., Herrera Padilla, Y., \& Martínez Torres, A. (2019). Propiedades Psicométricas de las versiones cortas del Cuestionario de Preocupación del Estado de Pensilvania. Universidad de la Laguna, 1 - 26. Recuperado el 03 de noviembre de 2021 ,

de https://riull.ull.es/xmlui/bitstream/handle/915/14622/Propiedades\%20psicometri cas\%20de\%20las\%20versiones\%20cortas\%20del\%20Cuestionario\%20de\%20Pr eocupacion\%20del\%20Estado\%20de\%20Pensilvania\%20\%28PSWQ\%29.pdf?s equence $=1 \&$ is Allowed $=\mathrm{y}$ 
Bados, A. (2009). Trastorno de Ansiedad Generalizada. Universitat de Barcelona, 1 - 98. $\begin{array}{lllllll}\text { Recuperado } & \text { el } & 11 & \text { de } & 11 & \text { de } & 2021,\end{array}$ http://diposit.ub.edu/dspace/bitstream/2445/6322/1/TAG.pdf

Borkovec, T. D., \& Inz, J. (1990). The nature of worry in generalized anxiety disorder: A predominance of thought activity. Behaviour Research and Therapy, 153 - 158. Recuperado el 15 de 11 de 2021

Dugas, M., Gosselin, P., \& Ladouceur, R. (2001). Intolerance of uncertainty and worry: Investigating specificity in a nonclinical sample. Cognitive Therapy and Research, 551-558. doi:http://dx.doi.org/0147-5916/01/1000-0551\$19.50/0.

Ferrer, A. (2002). La preocupación, mal de nuestros días. Psicología desde el Caribe(9), 76- 88. Recuperado el 15 de noviembre de 2021

Frenkel Brunswik, E. (1948). Intolerance of ambiguity as an emotional and perceptual personality variable. Journal of Personality, 108 - 143.

González Rodríguez, M., Cubas León, R., Rovella, A. T., \& Darias Herrera, M. (juliodiciembre de 2006). Adaptación española de la Escala de Intolerancia hacia la Incertidumbre: procesos cognitivos, ansiedad y depresión. Psicología y Salud, 16(2), 219 - 233. Recuperado el 03 de noviembre de 2021, de https://psicologiaysalud.uv.mx/index.php/psicysalud/article/view/775/1386

Hernández Sampieri, R., Fernández Collado, C., \& Baptista Lucio, M. D. (2014). Metodología de la investigación (sexta ed.). México: McGRAW-HILL / INTERAMERICANA EDITORES, S.A. DE C.V.

Johnson, M. C., Saletti Cuesta, L., \& Tumas, N. (junio de 2020). Emociones, preocupaciones y reflexiones frente a la pandemia del COVID-19 en Argentina. Ciência \& Saúde Coletiva, 2447 - 2456. doi: https://doi.org/10.1590/141381232020256.1 .10472020

Koerner, N., \& Dugas, M. J. (2008). An investigation of appraisals in individuals vulnerable toexcesive worry: The role of intolerance of uncertainty. Cognitive Therapy and Research, 619-638. Recuperado el 08 de noviembre de 2021, de http://dx.doi.org10.1007/s10608- 007-9125-2.

Medina, A. A. (Junio de 2020). LA COVID-19 - Transformación Educativa Obligada. Paraguay 2020. ACADEMIC DISCLOSURE UNA FENOB, 1(1), 40 - 51. 
Recuperado el 13 de noviembre de 2021, de https://revistascientificas.una.py/index.php/rfenob/article/view/103

Ministerio de Salud Pública y Bienestar Social. (2020). Presidencia de la República del Paraguay. Decreto 3576 de 2020, mayo 03 por el cual se establece Cuarentena Inteligente. MSP y $B S, 1$ - 9. doi:https://www.mspbs.gov.py/cuarentenainteligente.html

Padros Blazquez, F., Gonzalez Betanzos, F., Martinez Medina, M. P., \& Wagner, F. (2018). Propiedades Psicométricas del Cuestionario de Preocupación Pensilvania (PSWQ) de las Versiones Original y Reducida en Muestras Mexicanas. Actas Españolas Psiquiatría, 46(4), 117-124. Recuperado el 15 de noviembre de 2021

Rodríguez de Behrends, M., \& Brenilla, M. E. (2015). Adaptación para Buenos Aires de la Escala de Intolerancia a la Incertidumbre. INTERDISCIPLINARIA, Revista de Psicología y Ciencias Afines, 32(2), 261-274. Recuperado el 08 de noviembre de 2021, de https://www.redalyc.org/pdf/180/18043528004.pdf

Sanabria Benítez., R. S. (2020). Educación virtual debido a la pandemia del Covid-19 en Paraguay. Recuperado el 13 de 11 de 2021, de http://nuestravozacolores.org/educacion-virtual-debido-a-la-pandemia-delcovid-19-en-paraguay/

Tamayo y Tamayo, M. (2003). El proceso de investigación científica (cuarta ed.). México: LIMUSA, S.A. DEC.V.

Wehrle Martínez, A. (2020). Educación en contextos de COVID-19: requerimientos mínimos para una educación a distancia. Observatorio Educativo ciudadano. Recuperado el 13 de 11 de 2021, de Observatorio Educativo ciudadano.: https://www.observatorio.org.py/observatorio. 